\title{
Cryogenic Yb:YAG composite-thin-disk for high energy and average power amplifiers
}

\author{
Luis E. Zapata, ${ }^{1,3, *}$ Hua Lin, ${ }^{3}$ Anne-Laure Calendron ${ }^{1,2}$ Huseyin Cankaya, ${ }^{1,2}$, Michael Hemmer, ${ }^{1}$ Fabian \\ Reichert, ${ }^{1}$ W. Ronny Huang, ${ }^{3}$ Eduardo Granados, ${ }^{3,4}$ Kyung-Han Hong, ${ }^{3}$ and Franz X. Kärtner, ${ }^{1,2,3}$ \\ ${ }^{1}$ Center for Free-Electron Laser Science, Deutsches Elektronen-Synchrotron, Notkestrasse 85, 22607 Hamburg, Germany \\ ${ }^{2}$ Department of Physics and The Hamburg Centre for Ultrafast Imaging, Universität Hamburg, Luruper Chaussee 149, 22761 Hamburg, Germany \\ ${ }^{3}$ Department of Electrical Engineering and Computer Science and Research Laboratory of Electronics, \\ Massachusetts Institute of Technology (MIT), Cambridge, Massachusetts 02139, USA, \\ ${ }^{4}$ IKERBASQUE, Basque Foundation for Science, Bilbao, Spain \\ *Corresponding author: luis.zapata@cfel.de \\ Received Month X, XXXX; revised Month X, XXXX; accepted Month X, \\ XXXX; posted Month X, XXXX (Doc. ID XXXXX); published Month X, XXXX \\ A cryogenic composite-thin-disk amplifier with amplified spontaneous emission (ASE) rejection is implemented that \\ overcomes traditional laser system problems in high-energy pulsed laser drivers of high average power. A small signal gain \\ of $8 \mathrm{~dB}$ was demonstrated compared to $1.5 \mathrm{~dB}$ for an uncapped thin-disk without ASE mitigation under identical pumping \\ conditions. A strict image-relayed 12-pass architecture using an off-axis vacuum telescope and polarization switching \\ extracted $100 \mathrm{~mJ}$ at $250 \mathrm{~Hz}$ in high beam quality stretched $700 \mathrm{ps}$ pulses of 0.6 -nm bandwidth. \\ OCIS codes:(140.3280) Laser amplifiers; (140.3538) Lasers pulsed; (140.3480) Lasers, diode-pumped
}

High gain and uniform heat removal in a Nd:glass zigzag slab geometry for mitigation of thermo-optical effects with phase conjugation inside a strictly relayed multipass optical architecture have been central to the significant high mark in performance of the high energy pulsed (>25 J, $14 \mathrm{~ns}$ ), high average power $(150 \mathrm{~W})$ solid-state laser of diffraction-limited output in [1]. We are exploring simpler alternatives for scaling laser amplifiers utilizing thin-disk geometries, cryogenic cooling techniques and the better intrinsic material and spectroscopic properties found in modern $\mathrm{Yb}^{3+-}$ doped crystals for the generation of energetic ultrashort pulses at high repetition rates. The small quantum defect minimizes heat load while the availability of highbrightness diodes allows for pumping with high intensities. For average power scaling, the thin-disk geometry is a proven technique for enhanced cooling [2] as well as mitigation of thermo-optical effects. Room-temperature Yb:YAG and Yb:LuAG have emerged as the most successful and frequently used high-power laser materials in this geometry [3]. Output power in excess of $8.5 \mathrm{~kW}$ from a single thindisk in continuous wave operation has been reported albeit with reduced beam quality [4]. Also in reference [4], researchers at Trumpf Laser $\mathrm{GmbH}$ summarily report achieving a beam quality of $\mathrm{M}^{2} \sim 1.4$ in the laboratory at $4-\mathrm{kW}$ output from a single disk.

Advances in high average power with high beam quality have also come from operating $\mathrm{Yb}^{3+-}$ doped materials at liquid nitrogen temperature extending by nearly 100 -fold the useful output of rod-type amplifiers and demonstrating high-gain and low spatial distortion with excellent performance in the amplification of short pulses [5,6,7].

Here we combine the intrinsic advantages of the thin-disk geometry with the thermo-mechanical and thermo-optical leverage afforded by liquid nitrogen cooling $\mathrm{Yb}^{3+}$ :YAG crystals to enable high energy pulses at high average power and diffraction limited performance simultaneously. To achieve high pulse energy, which requires high gain, we are using a variant of the thin-disk: the composite-thin-disk (CTD) pioneered at LLNL [8, 9] that mitigates ASE, extending gain-storage performance and aperture scaling. The cryogenic CTD prototypes in these experiments had a $4.5-\mathrm{mm}$ aperture comprising a $1^{-}$ $\mathrm{mm}$ thick $10 \% \mathrm{Yb}$ :YAG disk optically bonded to a $4^{-}$ $\mathrm{mm}$ thick undoped YAG-crystal "cap" with shaped edges fashioned to eject fluorescence. In this Letter we report on gain-storage measurements with this CTD prototype compared with an uncapped disk under identical conditions. We also report on the extraction of $100 \mathrm{~mJ}$ chirped pulses at $250 \mathrm{~Hz}$ from a single CTD using a compact 12 -pass strictly relayed optical architecture. Our interest is scaling optical parametric chirped pulse amplifiers (OPCPAs) for applications requiring energetic pulses and high repetition rates such as high-flux high-harmonic generation [10]. Further scaling will use the 100-mJ class chirped pulse amplifier reported in this Letter as the first stage to larger Joule class systems.

At the heart of our laser driver is a diode pumped cryogenic CTD amplifier assembly depicted in Fig. 1. On the cooled face, the laser-grade high-reflector exists in thermal contact -through soldering- with liquid nitrogen cooled, heat-spreader. The opposite face of the disk shaped gain-volume is bonded to the index-matched "cap" of undoped YAG. The function of the cap is to dilute fluorescence diminishing the deleterious influence of ASE. The parabolic sidewalls have a smooth specular polish and efficiently eject 
fluorescence avoiding recirculation. The addition of a fully transparent cap does not affect the predominantly 1D thermal distribution in the gain volume, which in our finite-element thermal models was nearly identical in the CTD and uncapped disk. The undoped thermally insulated cap does not develop gradients rising to a uniform temperature, a benign effect that does not affect wavefront. The added thickness however results in a much stiffer gain element that is resilient to deformations [11].

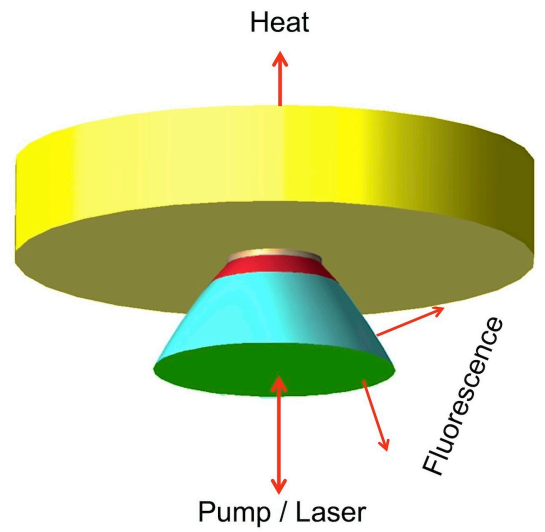

Fig. 1. A composite thin-disk (CTD) gain-element assembly is shown in this $3 \mathrm{D}$ model. The CTD $4.5-\mathrm{mm}$ clear aperture comprises a $1-\mathrm{mm}$ thick $10 \% \mathrm{Yb}: \mathrm{YAG}$ gain volume (red) bonded to a 4-mm thick undoped YAG "cap" (blue). The parabolic sides were designed with the aid of a MonteCarlo ray trace code to maximally eject fluorescence. The CTD is contacted thermally to the expansion-matched heat spreader (in yellow) via soldering. The backplane interfaces to the cold finger of a liquid nitrogen Dewar.

CTD and uncapped gain elements were soldered to respective heat spreaders then contacted with a compressed indium gasket to the cooling plate of a commercial Dewar to complete assembly. Boiling liquid nitrogen in the Dewar supplied primary cooling from the top and an anti-reflection (AR)coated vacuum window provided optical access to the gain element from below. A 500-W fiber-coupled diode laser (Laserline $\mathrm{GmbH}$ ) at $940 \mathrm{~nm}$ was initially used as the pump source. A customized optical package projected a $4.4-\mathrm{mm}$ diameter smooth, nearly-flattop pump profile $(<10 \%$ roll $)$ at a distance of $180 \mathrm{~mm}$. This image was cast onto the CTD through the AR coated front face at $13^{\circ}$ angle of incidence filling most of the $4.5-\mathrm{mm}$ diameter highreflection (HR) coated backplane. The diode pump bounced off the CTD backplane making a second pass through the gain volume and the unabsorbed pump was collected by a thermopile. The diodes were temperature tuned and the absorption optimized at $90 \%$ in this simple double-pass arrangement. Uncapped disks of dimensions and doping identical to the CTD's gain-volume were fabricated and tested to provide performance baselines. To ascertain that the designs had comparable energetics and thermal performance, we tested a CTD and an uncapped disk in free running mode. A stable linear resonator was configured $1-\mathrm{m}$ long arranged with a $30 \mathrm{~cm}$ lens at the midpoint and a flat $90 \%$ reflectivity output coupler. In tests, $495-\mathrm{W}$ diode pulses $1-\mathrm{ms}$ in duration pumped the gain elements at up to $300 \mathrm{~Hz}$. The uncapped disk and the CTD produced multimode beams that filled the aperture with comparable output averaging 68 and 72 Watts, respectively. We concluded from these data that under saturated (low) gain conditions, the CTD and uncapped disk perform equivalently.

The small signal or "storage" gain was measured directly after six passes (with the multipass arrangement described later in this Letter) using the expanded 4-mm-diameter, Gaussian-shaped 200-ps seed pulses from an $\mathrm{Yb}: \mathrm{KYW}$ regenerative amplifier (Amplitude Systemes S-Pulse ${ }^{\mathrm{TM}}$ ). To avoid saturating the gain, the beam was attenuated to $\sim 50 \mu \mathrm{J} / \mathrm{pulse}$ and pulse picked timing the arrival of the probe pulses at the end of "square" diode-pump pulses 1-ms in duration. The repetition rate of $20-\mathrm{Hz}$ was selected to avoid thermal effects. The small signal gain per "bounce" (or double-pass) was obtained as $2 \cdot \ln \left(G_{o}\right)=1 / 3$ $\cdot \ln \left(E_{p} / E_{i n}\right)$ where $E_{p}$ is the pulse energy after six gain passes and, $E_{\text {in }}$ the transmitted pulse energy measured identically under un-pumped conditions. The characteristic storage gain for the CTD and uncapped disk were dramatically different as can be seen in Fig. 2, which plots the double pass gain vs. stored energy per unit area using (1). The storage gain can be expressed as,

$$
\ln \left(G_{o}\right) \equiv \Delta N \sigma L=\frac{(1-Q D)}{h v / \sigma} \frac{P_{a b s} \tau}{\pi r^{2}}\left(1-e^{-t_{p} / \tau}\right)
$$

Here, the left hand corresponds to the experimental small signal gain followed by its (4-level) definition where $\Delta N$ is the inversion density at the end of the pump pulse, $\sigma$ the emission cross section and $L$ the gain-length along the probe dimension. In the right hand $\Delta N$ has been expanded to account for decay during the pump pulse and simplified to an expression based on the known or measurable quantities, where $\mathrm{QD}=9.1 \%$ is the quantum defect, $P_{a b s}$ is the measured absorbed power, $\tau=960 \mu$ s is the upper level lifetime, $t_{p}=1^{-} \mathrm{ms}$ is the diode-pump pulse duration, $h v$ is the laser-photon energy and $\pi r^{2}$ the pumped area of radius $r=2.2 \mathrm{~mm}$. In the absence of saturating effects the right hand in (1) is the energy stored in the upper laser level (ULL) per unit area divided by the saturation fluence $E_{s a t}=h v / \sigma$, linearly dependent on the applied pump power with slope equal to $1 / E_{\text {sat }}$.

As can be seen in figure 2, the double-pass gain rolls over reaching a limit of $8 \mathrm{~dB}$ at the highest 
pump power (the system was later upgraded with the installation of an analogous but more powerful $2-\mathrm{kW}$ diode pump unit from Laserline $\mathrm{GmbH}$ to extend the small signal gain performance). We attribute this evolution to the saturating effect of transverse ASE [11]. The initial slope in figure 2 yields the effective pulse saturation fluence $E_{\text {sat }}=3.5 \mathrm{~J} / \mathrm{cm}^{2}$.

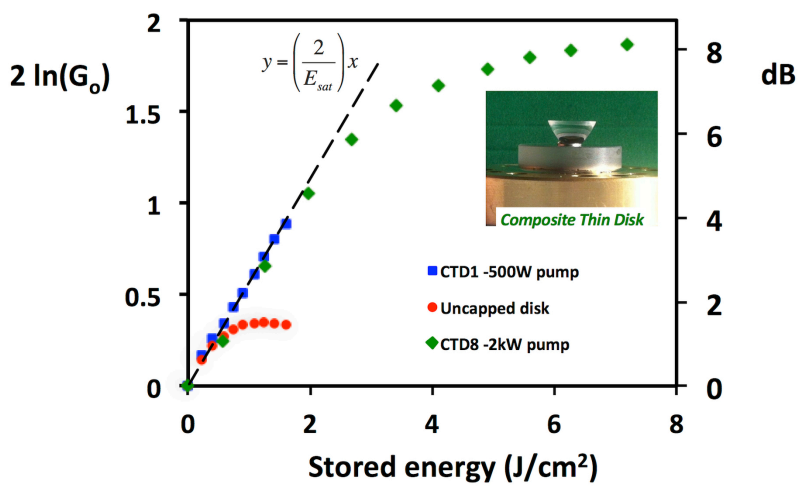

Fig. 2. The double-pass small-signal gain for an uncapped disk $(\bullet)$ and a composite thin disk (") were measured using a $500-\mathrm{W}$ diode pump at low duty factor. A $2-\mathrm{kW}$ pump was later installed extending the CTD performance to the ASE limit $(\bullet)$. The inset shows a CTD/ heat-spreader assembly.

This value is approximately twice the published value for narrow band operation at $100 \mathrm{~K}$ [5] and consistent with the $0.5 \mathrm{~nm}$ output linewidth (measured with an ANDO spectrometer) averaged over the 1-nm gain bandwidth at this temperature.
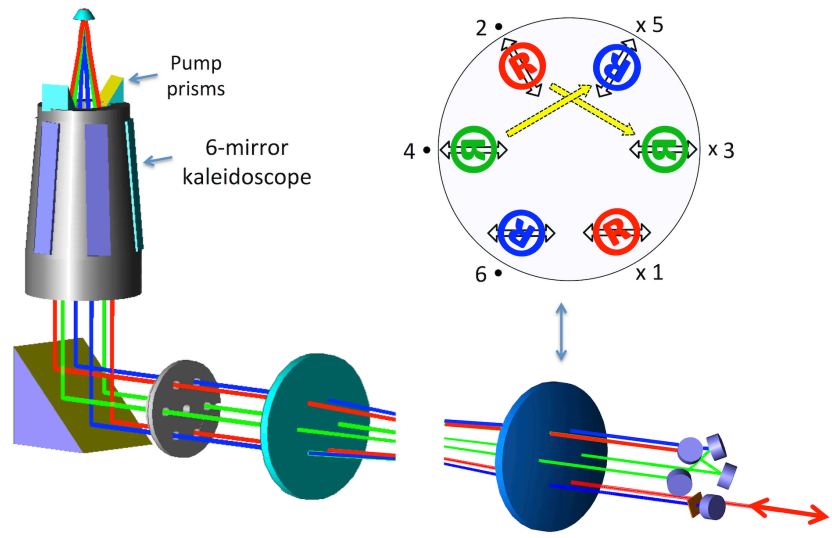

Fig. 3. Ray-trace of the strictly relayed 12-pass optical architecture used. The telescope is truncated in this diagram and the input/output TFP omitted for clarity. The insert on the upper right depicts the beam footprint; image and polarization rotation ( $\mathrm{R}$ and white arrow respectively).

To extract high pulse energy efficiently, we devised a strictly relayed 12 -pass architecture utilizing a 1:1 vacuum telescope off-axis. The optical components and beam passage is depicted in Fig. 3. The 12-pass hardware and elevated cryogenic Dewar containing the CTD can be seen in the picture, Fig. 4.
Two identical 3"-diameter, $f=300 \mathrm{~mm}$ planoconvex lenses doubling as vacuum windows were separated by the afocal distance $(2 \cdot f)$ set with the aid of a shear-plate during assembly. A formatted Gaussian P-polarized seed entered through a thinfilm polarizer (TFP) filling the 4-mm input/output aperture (in the lower right corner of Figs. 3 and 4). The seed entered the first lens off-center and parallel to the optical axis (OA) at the position of 5 o'clock (red trace in Fig. 3). At the other end of the telescope, a $45^{\circ}$ prism and six-mirror kaleidoscope (angled by $5^{\circ}$ ) deflected the seed towards the CTD reaching the $\mathrm{HR}$ at precisely the imaging distance of $4 \cdot f$ from the input/output aperture (a crosshair at the input aperture was imaged onto the CTD during alignment). The beam "bounced" off the CTD HR returning the amplified beam after two gain passes on a symmetric path. The beam exited diametrically opposed at the input lens (at 11 o'clock) and was deflected with two adjustable mirrors towards another off-axis entry point in the first lens (at 3 o'clock) repeating this pattern twice more (green trace and blue trace in Fig. 3) exiting at the position of 7 o'clock. Having passed the gain medium 6 times, the quarter wave plate and back mirror rotated the polarization from $\mathrm{P}$ to $\mathrm{S}$ returning the amplified beam for another 6 gain passes thus; the near field was strictly relayed in 12 gain passes through the CTD saturating the gain while becoming superGaussian. A 2-mm aperture in the vacuum telescope attenuated spatial features smaller than $0.3 \mathrm{~mm}$ insuring a smooth beam throughout for low damage risk. The round-trip transmission (per "bounce" or, two gain passes) measured $92 \%$.

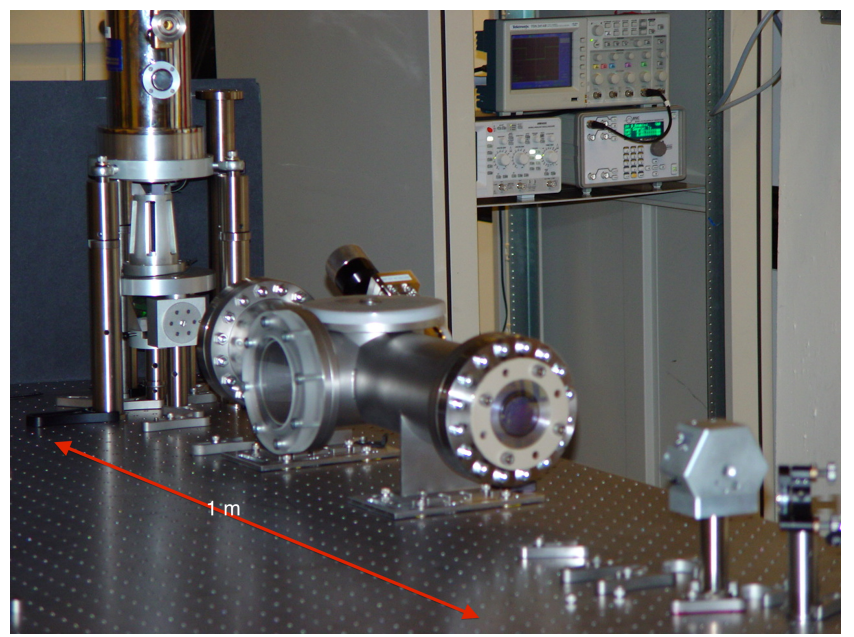

Fig. 4. The 12-pass hardware is shown in this picture. The CTD is mounted inside the liquid nitrogen Dewar and accessed from below. Precision-machined surfaces keyed the alignment of the optics.

Chirped pulse extraction yielded results presented in Figs. 5 and 6. In these experiments, the diode 
pump delivered square pulses of $250-\mu$ s duration at up to $2-\mathrm{kW}$, deemed as the best compromise between heat deposition and gain. Seeded with the $1.8-\mathrm{mJ}$, chirped 2.2-ns pulse from a homebuilt $\mathrm{Yb}: \mathrm{KYW}$ system described elsewhere [12], the CTD produced up to $110 \mathrm{~mJ}$ at $100 \mathrm{~Hz}$ with $35 \%$ slope and $26 \%$ overall optical efficiency. At higher repetition rates the slope decreased producing $100 \mathrm{~mJ}$ at $250 \mathrm{~Hz}$ with $22 \%$ slope, $21 \%$ overall optical efficiency. The farfield at the focus of a $\mathrm{f}=300-\mathrm{mm}$ lens was round with $1 / \mathrm{e}^{2}$ diameter that remained less than 1.1-timesdiffraction-limited when compared to the $2.44 \lambda / \mathrm{D}$ ideal central lobe expected if the near field $\left(\mathrm{D}=3.3^{-}\right.$ $\mathrm{mm}$ ) in Fig. 5 were uniformly illuminated. The farfield diagnostic showed the rapid onset of blooming as the average output power increased beyond $35 \mathrm{~W}$, the maximum reported in Fig. 5. The pointing and energy stability was measured at mid-power, averaging $\pm 20-\mu \mathrm{Rad}$ and $\pm 2.5 \%$ respectively over a 90-minute span.

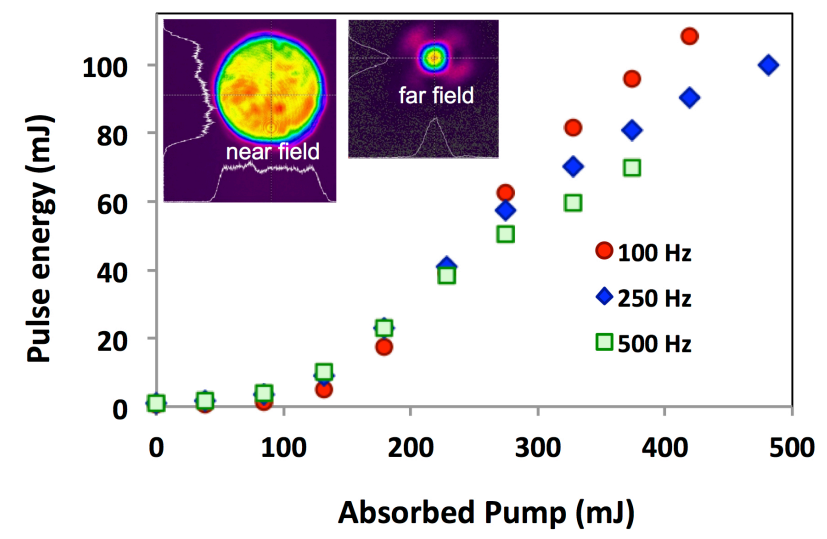

Fig. 5. The pulsed output reached a maximum of $110 \mathrm{~mJ}$ and slope efficiency of $48 \%$. Inset: near-field/far-field output profiles at $100 \mathrm{~mJ} / 250 \mathrm{~Hz}$.

The spectral overlap between the seed and gain profile was estimated at $\sim 0.66 \mathrm{~mJ}$ resulting in an inband signal gain greater than 100 at maximum pump. Measured spectra for the seed and, for the output at $20 \mathrm{~Hz}$ and $250 \mathrm{~Hz}$ are shown in Fig. 6. At $20 \mathrm{~Hz}$, the bandwidth at maximum gain was $0.47 \mathrm{~nm}$ however; at $250 \mathrm{~Hz}$, the bandwidth increased to 0.61 $\mathrm{nm}$ presumably due to the higher operating temperature within the gain medium. The output pulse width at maximum gain was 700 ps. The depolarization was rejected at the input Faraday isolator never exceeding $1.5 \%$ of the output power.

In conclusion, high gain was demonstrated with a newly designed Yb:YAG/YAG composite thin-disk operated cryogenically. A strictly relayed 12-pass optical architecture was used to extract $100 \mathrm{~mJ}$ chirped pulses at $250 \mathrm{~Hz}$ demonstrating the benefits afforded by the combination of these techniques for high energy and high average power. We are planning on larger CTDs and aggressive cooling technologies for higher performance $(1-\mathrm{J}$ at $1-\mathrm{kHz})$.

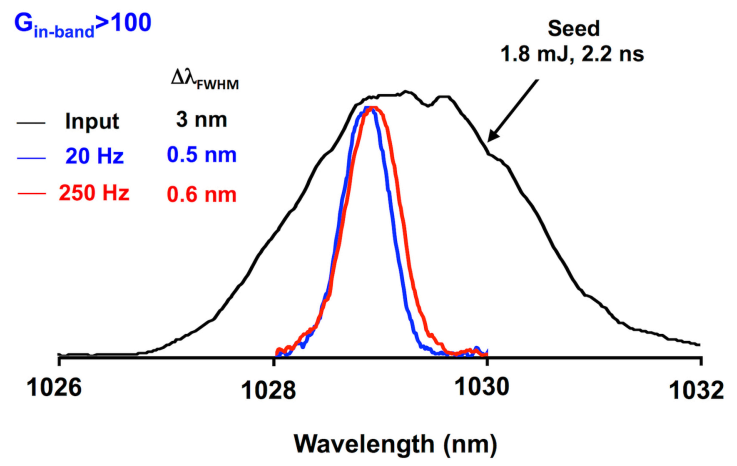

Fig. 6. The measured seed spectrum and, gain-narrowed output spectra at $100-\mathrm{mJ}$ output for $20 \mathrm{~Hz}$ and $250 \mathrm{~Hz}$ are compared. The vertical scale is in arbitrary units.

We appreciate the many helpful discussions with T. Y. Fan, Darren Rand, Leo Missaggia and Michael Brattain of MIT-Lincoln Laboratory and their professional assistance with indium bonding technology. We further thank Andrew Ryan and Andrew Gallant of the MIT machine shop, Lars Gumprecht of DESY and their engineering support teams. This work was supported through the DESYMIT Collaboration and "The Hamburg Centre for Ultrafast Imaging- Structure, Dynamics and Control of Matter at the Atomic Scale" of the Deutsche Forschungsgemeinschaft.

\section{References}

[1] C. B. Dane, L. E. Zapata, W. A. Neuman, M. A. Norton, and L. Hackel, IEEE JQE 31, 148 (1995)

[2] A. Giesen and J.Speiser, JSTQE13, 598(2007)

[3] K. Beil, S. T. Fredrich-Thornton, F. Tellkamp, R. Peters, C. Kränkel, K. Petermann, and G. Huber, Opt. Express 18, 20712 (2010)

[4] T. Gottwald, V. Kuhn, S.-S. Schad, C. Stolzenburg, and A. Killi, Proc. SPIE 8898, Technologies for Optical Countermeasures X; and High-Power Lasers 2013: Technology and Systems, 88980P (October 15, 2013)

[5] T. Y. Fan, D. J. Ripin, R. L. Aggarwal, J. R. Ochoa, B. Chann, M. Tilleman, and J. Spitzberg, JSTQE 13, 448-453, (2007)

[6] D. E. Miller, L. E. Zapata, D. J. Ripin, and T. Y. Fan, Optics Letters 37 (13), 2700, (2012)

[7] K.-H. Hong, J. Gopinath, D. Rand, A. Siddiqui, S.-W. Huang, E. Li, B. J. Eggleton, J. Hybl, T. Y. Fan, and F. X. Kärtner, Opt. Lett. 35, 1752 (2010)

[8] S. A. Payne; W. F. Krupke; R. J. Beach; S. B. Sutton; E. C. Honea; C. Bibeau; H. Powel. "High average power scaleable thin-disk laser". U. S. patent. 6347109 (2002)

[9] L. E. Zapata, "Edge-Facet Pumped, multi-aperture, thindisk laser geometry for very high Average Power Output Scaling" U. S. Patent 6,834,070 B2, (2004)

[10] K.-H. Hong, C.-J. Lai, J. Siqueira, P. Krogen, J. Moses, C.L. Chang, G. J. Stein, L. E. Zapata and F. X. Kärtner, Opt. Lett. 39, 3145-3148 (2014)

[11] J. Speiser, J. Opt. Soc. Am. B/Vol. 26, No. 1/January 2009

[12] A. L. Calendron, H. C,ankaya and F. X. Kärtner OE Vol. 22 Issue 20, 24752-24762 (2014) 


\section{Full References}

[1] C. B. Dane, L. E. Zapata, W. A. Neuman, M. A. Norton and L. Hackel, "Design and Operation of a 150 Watt Near Diffraction-Limited Laser Amplifier with SBS Wave front Correction", IEEE Journal of Quantum Electronics, Vol. 31, No. 1, January 1995.

[2] Adolf Giesen and JochenSpeiser, "Fifteen Years of Work on Thin-Disk Lasers: Results and Scaling Laws", IEEE JOURNAL OF SELECTED TOPICS IN QUANTUM ELECTRONICS, VOL. 13, NO. 3, MAY/JUNE 2007

[3] K. Beil, S. T. Fredrich-Thornton, F. Tellkamp, R. Peters, C. Kränkel, K. Petermann, and Günter Huber, "Thermal and laser properties of Yb:LuAG for $k W$ thin disk lasers", OPTICS EXPRESS, 18, 20712 (2010)

[4] T. Gottwald, V. Kuhn, S.-S. Schad, C. Stolzenburg, and A. Killi, "Recent developments in high power thin disk lasers at TRUMPF Laser" Proc. SPIE 8898, Technologies for Optical Countermeasures X; and High-Power Lasers 2013: Technology and Systems, 88980 (October 15, 2013)

[5] T. Y. Fan, D. J. Ripin, R. L. Aggarwal, J. R. Ochoa, B. Chann, M. Tilleman, and J. Spitzberg, "Cryogenic Yb3+ -Doped Solid-State Lasers," IEEE J. Sel. Topics in Quant. Electron. 13 (2007) 448-453

[6] D. E. Miller, L. E. Zapata, D. J. Ripin, and T. Y. Fan, "Sub-picosecond pulses at 100-W average power from a Yb:YLF chirped-pulse amplification system" OPTICS LETTERS / Vol. 37, No. 13, 2700 / July 1, 2012

[7] K.-H. Hong, J. Gopinath, D. Rand, A. Siddiqui, S.-W. Huang, E. Li, B. J. Eggleton, J. Hybl, T. Y. Fan, and F. X. Kärtner, "High-energy, kHz-repetition-rate, ps cryogenic Yb:YAG chirped-pulse amplifier," Opt. Lett. 35, 1752 (2010).

[8] Stephen A. Payne; William F. Krupke; Raymond J. Beach; Steven B. Sutton; Eric C. Honea; Camille Bibeau; Howard Powel (2002). "High average power scaleable thin-disk laser". US patent. 6347109 with the University of California

[9] L. E. Zapata "Edge-Facet Pumped, multi-aperture, thin-disk laser geometry for very high Average Power Output Scaling" U. S. Patent 6,834,070 B2 December 21, 2004. under DOE contract No. W-7405-ENG-48 with the University of California

[10] K.-H. Hong, C.-J. Lai, J. Siqueira, P. Krogen, J. Moses, C.-L. Chang, G. J. Stein, L. E. Zapata, and F. X. Kärtner, "Multi-mJ, kHz, 2.1-mm optical parametric chirped pulse amplifier and high-flux soft X-ray high-harmonic generation," Opt. Lett. 39, 3145-3148 (2014).

[11] J Speiser, "Scaling of thin-disk lasers-influence of amplified spontaneous emission", Opt. Soc. Am. B/Vol. 26, No. 1/ (2009)

[12] A. L. Calendron, H. Çankaya and F. X. Kärtner, High-energy kHz Yb:KYW dual-crystal regenerative amplifier, OE Vol. 22 Issue $20,24752-24762(2014)$ 